\title{
Desarrollo temprano del roble (Nothofagus obliqua): un análisis arquitectural de procedencias de Argentina
}

\author{
Early development of roble (Nothofagus obliqua): \\ an architectural analysis of provenances from Argentina
}

\section{Javier G. Puntieri ${ }^{1 *}$, Javier E. Grosfeld ${ }^{1}$, Marina Stecconi ${ }^{1}$, Cecilia Brion ${ }^{1}$, María Marta Azpilicueta ${ }^{2}$, Leonardo Gallo ${ }^{2}$}

\footnotetext{
* Autor de correspondencia: ${ }^{1}$ Universidad Nacional del Comahue, Departamento de Botánica, CONICET, Quintral 1250, 8400 Bariloche, Argentina, tel. 54- 2944423374, fax: 54- 2944422111, jpuntier@crub.uncoma.edu.ar

${ }^{2}$ Instituto Nacional de Tecnología Agropecuaria (INTA), Unidad de Genética Forestal, Bariloche, Argentina.
}

\begin{abstract}
SUMMARY
The architectural development of three-year-old seedlings of Nothofagus obliqua under nursery conditions was studied. The seedlings were grown from seeds collected from five provenances within the natural distribution area of this species in Argentina. The length, number of nodes and basal diameter of the main axis' annual shoots were registered for 40 or more seedlings per provenance. The first-year shoot of all seedlings consisted on one extension unit (EU); second-year shoots had one or two EU and third-year shoots one, two or three EU. The development of a second or a third EU always resulted from the death of the apex of the first or second EU respectively. The most evident consequences of the existence of more than one EU in one year were: (a) greater length and number of nodes per shoot in that year, (b) a thicker stem at ground level and (c) a longer extension period. Plants from different provenances differed in the length of the first-year shoot and the proportion of second- and third-year shoots with one or more than one EU.
\end{abstract}

Key words: intraspecific variation, extension units, seedling, morphology, shoot.

\section{RESUMEN}

Se estudió el desarrollo arquitectural en tres años de plántulas de Nothofagus obliqua bajo condiciones de vivero. Las plántulas derivaron de semillas de cinco procedencias dentro del área de distribución natural de esta especie en Argentina. En no menos de 40 plantas por procedencia se registraron: longitud, número de nudos y diámetro basal de los brotes anuales del eje vertical principal. El brote del primer año de todas las plántulas consistió en una sola unidad de alargamiento (UA), el del segundo año en una o dos UA y el del tercero en una, dos o tres UA. El desarrollo de dos o tres UA resultó invariablemente de la muerte del meristema apical luego de la primera UA. Las consecuencias más evidentes de la existencia de más de un evento de alargamiento en un año fueron: (a) mayores longitud y número de nudos en ese año, (b) mayor engrosamiento del tronco al nivel del suelo y (c) un período de alargamiento más largo. Plantas de diferente procedencia difirieron en la longitud del brote del primer año y en las proporciones de brotes de los años siguientes con una o varias UA.

Palabras clave: variación intraespecífica, unidades de extensión, plántula, morfología, brote.

\section{INTRODUCCIÓN}

Los bosques templados del sur de Argentina y Chile cubren un amplio gradiente latitudinal y altitudinal a ambos lados de la Cordillera de los Andes. El género Nothofagus (familia Nothofagaceae) incluye a los principales componentes arbóreos de estos bosques. Nothofagus obliqua (Mirb.) Oerst., conocido como roble o roble pellín, es una especie forestal con alto valor ecológico y económico (Donoso 1993). Se trata de una especie caducifolia, anemófila y anemocora, que puede alcanzar 35-
$40 \mathrm{~m}$ de altura y hasta 1-2 m de diámetro del tronco. En Chile ocupa una amplia extensión que abarca desde la Cordillera de la Costa hasta la cordillera de Los Andes (entre $33^{\circ}$ y $41^{\circ} 30^{\prime} \mathrm{S} ; 1$ ), mientras que en Argentina presenta una distribución más restringida y fragmentada (entre 3649' y 40¹1' S; Donoso 1993, Gallo et al. 2000), principalmente dentro del Parque Nacional Lanín. La distribución de esta especie en Argentina sigue principalmente los valles postglaciarios transversales a los Andes en sentido oeste-este, en concordancia con las respectivas cuencas lacustres (Donoso et al. 2004). En las últi- 
mas décadas, sus bosques han sido muy afectados por actividades antrópicas.

Su limitada distribución natural en Argentina, el alto riesgo de degradación de sus poblaciones y sus atributos como especie forestal hacen del roble una especie relevante para el desarrollo de políticas de conservación y la implementación de planes de conservación y mejoramiento genético, como los que se encuentran en desarrollo tanto en Chile (por ejemplo, Emhart et al. 2000) como en Argentina (Gallo et al. 2000).

El estudio de la variación genética dentro de una especie vegetal puede realizarse a través de marcadores genéticos o moleculares, o a partir de la evaluación de caracteres morfométricos, fenológicos o fisiológicos en ensayos de procedencias y pruebas de progenies, que constituyen la esencia de los programas de mejoramiento genético. La evaluación precoz de parámetros o criterios de selección tales como el vigor germinativo o el crecimiento en altura de plántulas de diferentes procedencias o progenies es relevante en programas de mejoramiento genético con fines forestales, ya que contribuye en la toma de decisiones y acorta los plazos de mejoramiento (Ipinza 2000). Sin embargo, los atributos morfométricos usualmente tomados en cuenta en la selección de individuos para forestaciones (por ejemplo, altura total, número de ramas y diámetro de la base del tronco) suelen no expresar fielmente las diferencias genéticas entre plantas. Los vínculos entre variación genética y atributos morfológicos pueden evidenciarse mediante la aplicación de técnicas de análisis arquitectural desarrolladas en las últimas décadas (Hallé et al. 1978).

En el análisis arquitectural, el crecimiento de una planta es interpretado como la expresión de procesos endógenos que determinan su desarrollo y que se manifiestan mediante secuencias de eventos generadas principalmente en el funcionamiento coordinado de sus meristemas. El análisis arquitectural plantea una visión global, detallada e integradora entre la estructura y la dinámica de desarrollo de los vegetales. Constituye un sistema conceptual y metodológico que permite llegar a una síntesis del modo de desarrollo de una especie partiendo del estudio de la morfología y la disposición espacial de las unidades estructurales de su sistema caulinar (Hallé et al. 1978, Barthélémy et al. 1989, 1997). En este tipo de análisis se destacan las características morfológicas compartidas por los ejemplares de una especie. Sin embargo, a fin de explicar la totalidad de la estructura final de un árbol es necesario abordar tanto las variaciones debidas a factores externos como aquellas que responden a diferencias genéticas entre individuos (e.g. Costes et al. 1995). $\mathrm{El}$ análisis arquitectural permite analizar estas dos influencias al estudiar plantas de origen conocido o genéticamente diferenciadas y cultivadas bajo condiciones homogéneas (Guédon et al. 2003).

El análisis de la variabilidad morfológica y arquitectural de especies sudamericanas del género Nothofagus ha sido abordado recientemente (Passo et al. 2002, Puntieri et al. 1998, 1999, 2000, 2001ab, 2002, Souza et al. 2000, Stecconi et al. 2000). Partiendo del conocimiento de las influencias genéticas y ambientales sobre la arquitectura individual, generado en estos estudios, se pueden definir pautas de selección para el manejo de bosques naturales. Asimismo, estos estudios permiten mejorar nuestra comprensión de los patrones de distribución de estas especies a través del análisis del efecto limitante de factores ambientales. No obstante, tanto para el roble como para el raulí (Nothofagus alpina), las especies de Nothofagus forestalmente más relevantes y bajo mayor presión de explotación, la información arquitectural es escasa (Raffaele et al. 1998, Barthélémy et al. 1999) y se desconoce el grado de variación arquitectural tanto dentro de cada población natural como entre poblaciones.

Los objetivos del presente trabajo fueron: estudiar la variación arquitectural en el desarrollo inicial de plantas de roble de diferentes procedencias y analizar la posibilidad de adoptar determinadas características morfológicas y arquitecturales como criterios de selección temprana para programas de mejoramiento genético de esta especie.

\section{MÉTODOS}

Se analizó el desarrollo de plántulas de 3 años de edad en un ensayo de procedencias de roble instalado en el vivero de la Unidad de Genética Forestal de la Estación Experimental Agropecuaria del Instituto Nacional de Tecnología Agropecuaria (INTA), Bariloche, Argenti-

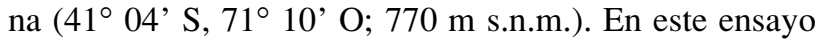
se analizó el desarrollo de plántulas derivadas de semillas generadas por polinización espontánea y recolectadas en cinco procedencias de cuatro áreas geográficas de Argentina, denominadas según la localidad de origen de las semillas: Norquinco, Quillén, Pilolil y Quila-Quina (cuadro 1). Las semillas fueron cosechadas en el año 2000 y sembradas en contenedores en la primavera de ese año. Al año siguiente las plantas se instalaron en el dispositivo experimental, con suelo forestal, a la intemperie bajo media sombra y riego por aspersión. Este dispositivo consistió en tres bloques completos en cada uno de los cuales se ordenó aleatoriamente un grupo de entre 15 y 20 plántulas de cada procedencia (ese número dependió de la disponibilidad de plántulas de las distintas procedencias). Las plántulas de cada grupo fueron dispuestas en hileras, con una distancia entre plántulas de aproximadamente $15 \mathrm{~cm}$, lo que garantiza el espacio necesario para su crecimiento hasta su salida hacia los ensayos de campo. Los bloques estuvieron distanciados a $50 \mathrm{~cm}$ y se dispuso una hilera de plántulas de roble de la misma edad (no incluidas en las parcelas) a modo de borde alrededor de cada bloque. El diseño experimental fue de bloques completos al azar, un diseño estadístico que permite considerar la distribución de los bloques sobre 
Cuadro 1. Coordenadas y altitud de las zonas de procedencia de las semillas utilizadas para las poblaciones experimentales del presente estudio.

Coordinates and altitude of the provenance sites of the seeds used for the experimental populations of the present study.

\begin{tabular}{lclc}
\hline Procedencia & $\begin{array}{c}\text { Latitud } \\
(\mathrm{S})\end{array}$ & $\begin{array}{c}\text { Longitud }) \\
(\mathrm{O})\end{array}$ & $\begin{array}{c}\text { Altitud } \\
(\mathrm{m} \text { s.n.m. })\end{array}$ \\
\hline Norquinco & $39^{\circ} 09^{\prime} 11^{\prime}$, & $71^{\circ} 15^{\prime}, 03^{\prime}$, & 1.071 \\
Quillén 7 & $39^{\circ} 22^{\prime} 16^{\prime}$, & $71^{\circ} 17^{\prime}, 31^{\prime}$, & 1.140 \\
Quillén 8 & $39^{\circ} 26^{\prime} 18^{\prime}$, & $71^{\circ} 25^{\prime} 37^{\prime}$, & 1.100 \\
Pilolil & $39^{\circ} 30^{\prime} 05^{\prime}$, & $70^{\circ} 57^{\prime}, 44^{\prime}$, & 836 \\
Quila-Quina & $40^{\circ} 10^{\prime} 40^{\prime}$, & $71^{\circ} 26^{\prime} 37^{\prime}$, & 983 \\
\hline
\end{tabular}

el terreno (en nuestro caso canteros de cultivo) y las probables fuentes de variación ambiental dentro del vivero (en nuestro caso esencialmente sombra y riego) y cuantificar esas fuentes de variación en el análisis estadístico. Se realizaron mediciones sobre todas las plantas de las cinco procedencias que de aquí en adelante denominaremos: Ñorquinco, Quillén 7, Quillén 8, Pilolil y QuilaQuina (cuadro 1).

En octubre de 2002 se evaluó el crecimiento de cada plántula en los primeros 2 años de desarrollo (períodos 2000-2001 y 2001-2002) y en el invierno de 2003 su crecimiento en el período 2002-2003. Para el eje vertical principal de cada planta se identificaron los límites morfológicos de cada porción de eje resultante de un evento ininterrumpido de alargamiento, de aquí en adelante denominada unidad de alargamiento (UA), y de cada porción de eje resultante del alargamiento de ese eje en un año, que en adelante denominaremos brote (Barthélémy et al. 1999). Se registraron: longitud, número de nudos, diámetro basal y condición del ápice (vivo o muerto) de cada UA de cada brote (figura 1).

Luego de la medición de 2003 se registraron, para cada planta, la longitud total y el número de nudos total del tronco (sumando los valores correspondientes a los tres brotes producidos), así como el diámetro basal del tronco y los cocientes entre longitud total, número de nudos total y diámetro basal del tronco.

Las comparaciones de longitud, número de nudos y diámetro de las UA se realizaron mediante análisis de la variancia de un factor (ANDEVA y prueba $F$ de Fisher). Las comparaciones de las características del tronco luego de tres años de desarrollo (longitud total, número de nudos total, diámetro basal y los cocientes entre estas variables) se realizaron con ANDEVA, incluyendo como factores la procedencia y el número de UA de los brotes 2 o 3 . Las comparaciones de medias después de ANDEVA se realizaron con tests a posteriori de Tukey. Se compararon las proporciones de brotes con ápice muerto y las de brotes constituidos por más de una UA mediante pruebas de chi-cuadrado ( $\chi^{2}$; Sokal y Rohlf 1981).

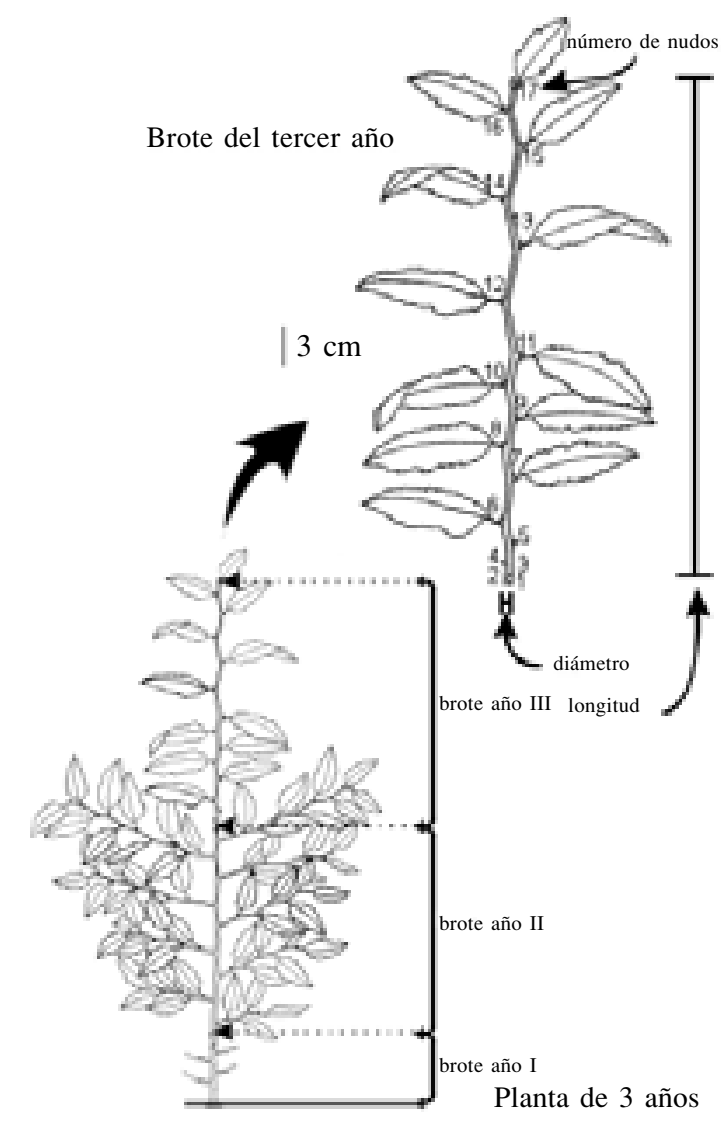

Figura 1. Ilustración semiesquemática de la porción aérea de una planta de roble de 3 años. Cada brote anual consistió en una unidad de alargamiento. Con flechas punteadas horizontales se indican los límites entre los brotes anuales. Sobre un detalle del tercer brote de la misma planta se indican las tres variables del crecimiento obtenidas para cada brote: número de nudos, longitud y diámetro basal. De las ramas derivadas del brote del primer año sólo se ilustró la porción basal.

Semi-diagrammatic representation of the aerial portion of a three-year-old oak. Each shoot consisted of one extension unit. Horizontal dashed arrows indicate between-shoot limits. The three variables registered for each shoot, i.e. number of nodes, length and diameter, are indicated in a detailed third-year shoot. Only the proximal end of the branches derived from the first-year shoot is drawn.

\section{RESULTADOS}

Tamaño de los brotes en años sucesivos. En todas las plantas fueron evidentes los marcadores morfológicos de las detenciones del crecimiento primario entre brotes y entre UA dentro de un brote. El brote producido en el primer año desde la germinación fue el de menor tamaño en todas las plantas (con promedios de $10,5 \mathrm{~cm}$ y 11 nudos; figura 2) y consistió en una sola UA. Se encontraron diferencias significativas entre procedencias en la longitud del brote del primer año $(F=12,6, P<0,001)$ pero no en su número de nudos ni en su diámetro medido 
al finalizar el segundo año $(F \leq 2,0, \mathrm{P}>0,05$; figura 2$)$. El brote del primer año presentó longitud significativamente menor para las plantas de las procedencias Norquinco y Quila-Quina que para las de las procedencias Pilolil, Quillén 7 y Quillén 8.

El porcentaje de brotes del primer año con ápice muerto al final de ese período de alargamiento fue mayor al $70 \%$ en cada una de las procedencias (figura 3). Para las procedencias Norquinco y Pilolil se detectó menor proporción de ápices muertos que para las procedencias restantes $\left(\chi^{2}=9,9 ; P<0,05\right)$. El brote del segundo año derivó, en la mayoría de los casos, de una yema axilar del brote del primer año.
El brote del segundo año se desarrolló en una o en dos UA, y en este último caso se registró la muerte del ápice de la primera UA y la formación de un relevo a partir de una yema axilar distal. No obstante, en algunos brotes la muerte del ápice durante el desarrollo de la primera UA no resultó en el desarrollo de una segunda UA. La proporción de brotes del segundo año con dos UA fue menor para las dos procedencias de Quillén $(\sim 13 \%)$ que para las restantes tres procedencias $(\sim 29 \%$; $\chi^{2}=9,9 ; P<0,05$; cuadro 2). La longitud, el diámetro basal y el número de nudos de cada una de las UA de los brotes del segundo año fueron similares para todas las procedencias $(F \leq 2,4, P>0,05$; figura 2$)$. La longitud y
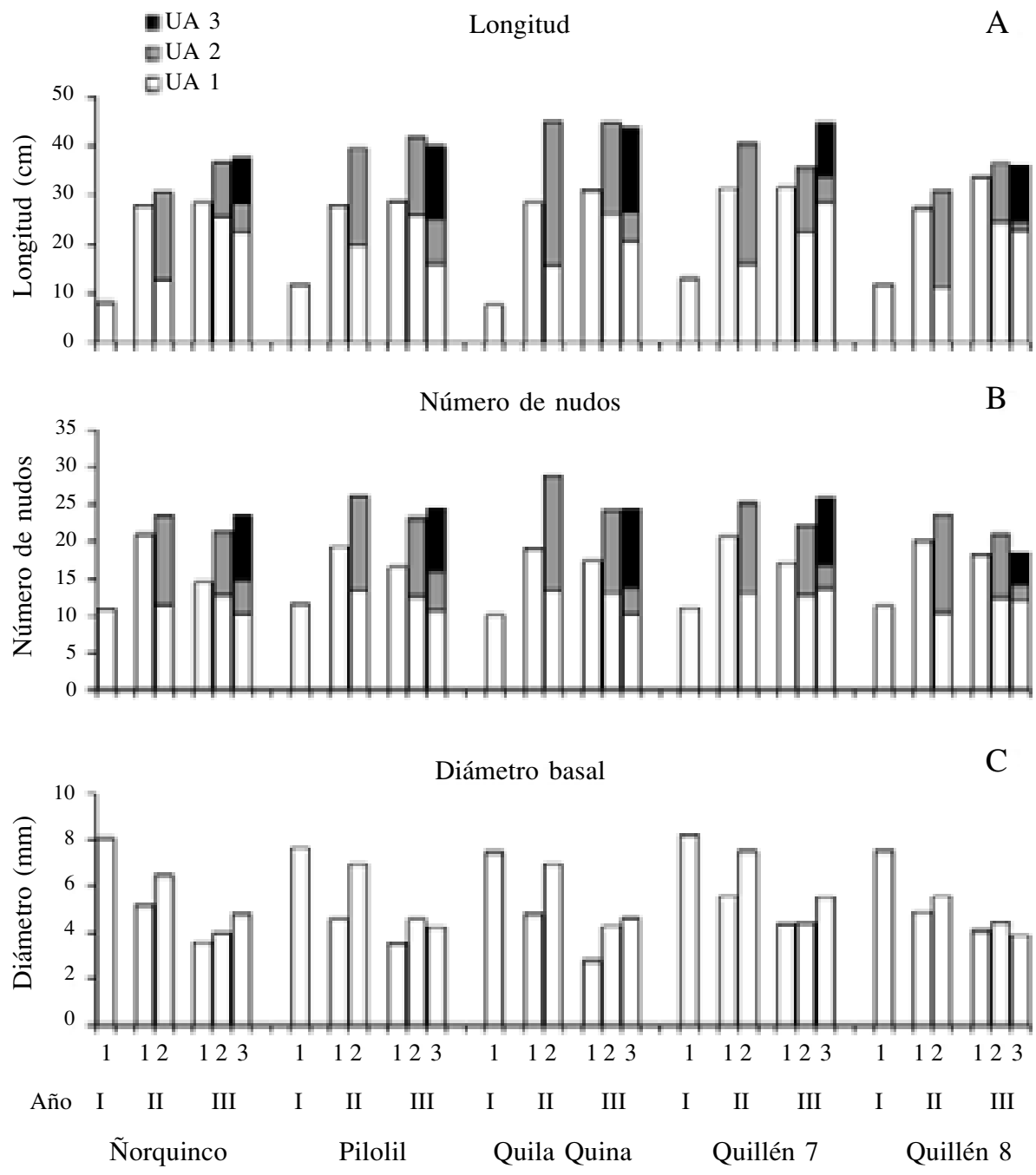

Figura 2. Promedio de la longitud (A), el número de nudos (B) y el diámetro basal (C) de brotes con 1, 2 o 3 unidades de alargamiento (UA) de los tres primeros años de desarrollo (I, II y III) de plantas de roble de cinco procedencias. Se indican las longitudes y números de nudos de las UA 1,2 y 3 de los brotes con 2 o 3 UA.

Mean length (A), number of nodes (B) and basal diameter (C) of shoots with 1, 2 or 3 extension units (UA) developed by oak plants of each provenance in their first three years (I, II and III). The length and number of nodes of UA 1, 2 and 3 of shoots with 2 or 3 UA are indicated. 


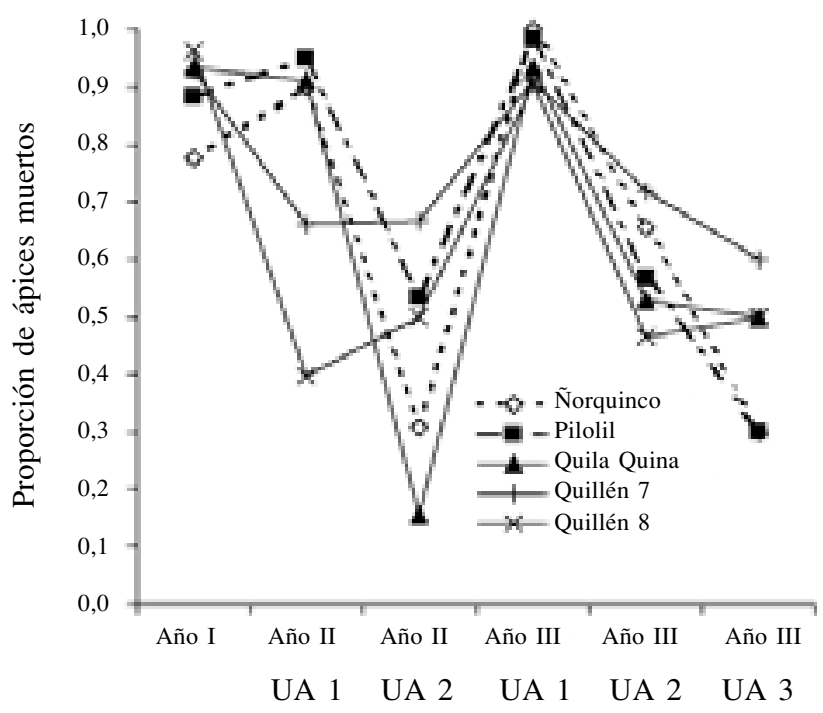

Figura 3. Proporción de unidades de alargamiento (UA) con el ápice muerto luego de su alargamiento para brotes de roble de diferentes años (I, II y III) y procedencias.

Proportion of extension units (UA) with apex death after extension for shoots of oak of different years (I, II and III) and provenances.

el número de nudos de la primera UA de los brotes del segundo año con dos UA fueron menores que los de brotes de una UA ( $F=39,2$ para la longitud y $F=64,4$ para el número de nudos; $P<0,001$; figura 2 ). La longitud, el diámetro basal y el número de nudos de brotes del segundo año con dos UA fueron mayores que los de brotes de ese año con una UA $(F=15,1, F=33,9$ y $F=33,0$, respectivamente; $P<0,001$; figura 1$)$.

Para la primera UA del segundo año, el porcentaje de ápices muertos fue elevado aunque, en promedio, fue menor que para el primer año debido a la significativamente mayor sobrevivencia de ápices de las procedencias de Quillén ( $\chi^{2}=61,1 ; P<0,001$; figura 3). En el caso de la segunda UA de los brotes del segundo año, la proporción de ápices muertos fue, en comparación con la primera UA de ese año, menor en las procedencias Norquinco, Pilolil y Quila-Quina y similar en las procedencias de Quillén.

El brote del tercer año se desarrolló en una, dos o tres UA. La segunda y tercera UA se desarrollaron luego de la muerte del ápice de la primera o de la segunda UA del mismo brote, respectivamente. Para ambas procedencias de Quillén, hubo mayor proporción de plantas con el tercer brote constituido por una sola UA $(\sim 44 \%)$ que para las restantes procedencias $\left(\sim 21 \% ; \chi^{2}=30,8\right.$; $P<0,001$; cuadro 2). La proporción de brotes con tres UA fue baja para todas las procedencias, pero algo superior para Ñorquinco (17\%). No se registraron diferencias entre procedencias para la longitud, el diámetro y el número de nudos de los brotes del tercer año (sumando
Cuadro 2. Porcentaje de brotes anuales del segundo y tercer años conformados por una, dos o tres unidades de alargamiento (UA) en robles de diferentes procedencias.

Percentage of second- and third-year annual shoots consisting of one, two or three extension units (UA) for oak of different provenances.

Número de unidades de alargamiento (UA) por brote anual

\begin{tabular}{lccc}
\cline { 2 - 3 } & 1 UA & 2 UA & $3 \mathrm{UA}$ \\
\hline Año 2 & & & \\
$\quad$ Ñorquinco & 67,5 & 32,5 & 0 \\
Pilolil & 74,6 & 25,4 & 0 \\
Quila-Quina & 71,1 & 28,9 & 0 \\
Quillén 7 & 89,1 & 10,9 & 0 \\
Quillén 8 & 84,9 & 15,1 & 0 \\
& & & \\
Año 3 & & & \\
Ñorquinco & 27,5 & 47,5 & 25,0 \\
Pilolil & 13,6 & 76,3 & 10,2 \\
Quila-Quina & 24,4 & 66,7 & 8,9 \\
Quillén 7 & 44,6 & 39,3 & 16,1 \\
Quillén 8 & 43,4 & 52,8 & 3,8 \\
\hline
\end{tabular}

todas las UA para la longitud y el número de nudos; $F \leq 2,0 ; P>0,05$; figura 2 ). La longitud y el número de nudos de los brotes con una UA fueron mayores que los de la primera UA de brotes con más de una UA $(F=7,8$ y 25,3 , respectivamente; $P<0,005)$ pero menores que los de las sumas de las longitudes y los números de nudos de todas las UA de estos últimos brotes $(F=7,7$ y 23,4 , respectivamente; $P<0,005$; figura 2 ). En ninguno de estos casos se registraron diferencias significativas entre las primeras UA de brotes con dos y con tres UA (prueba de Tukey). La longitud y el número de nudos de la segunda UA en brotes del tercer año con dos UA fueron mayores que los de la segunda UA de brotes con tres UA $(F=19,0$ y 53,4 , respectivamente; $P<0,001)$. El diámetro basal de brotes con una UA después de su alargamiento fue menor que el de brotes con varias UA $(F=5,9 ; P<0,005)$.

Para la primera UA del tercer año, la muerte del ápice fue muy frecuente para todas las procedencias, aunque las de Quillén y Quila-Quina presentaron valores algo inferiores. En el caso de la segunda UA del tercer año, el porcentaje de ápices muertos disminuyó con respecto al de la primera UA de ese año. Para la tercera UA del tercer año, la proporción de ápices muertos fue, en promedio, algo inferior a la observada para la segunda UA de ese año. Para ninguna de las tres UA del tercer año se detectaron diferencias significativas entre procedencias con respecto a la proporción de brotes con ápice muerto $\left(\chi^{2}=5,6 ; 5,1\right.$ y 1,6 , respectivamente, para las UA primera, segunda y tercera; figura 3 ). 
Se registraron diferencias entre procedencias en la longitud total del tronco al cabo de los tres períodos de crecimiento al incorporar en el análisis el número de UA del segundo brote (cuadro 3): las plantas de las procedencias Ñorquinco y Quillén 8 presentaron troncos más cortos que las de las procedencias Pilolil, Quila-Quina y Quillén 7. Los diámetros basales difirieron entre procedencias al incorporar en el análisis el número de UA del tercer brote (Quila-Quina < Quillén 7). La longitud y el número de nudos del tronco y su diámetro basal al cabo de tres períodos de crecimiento fueron significativamente afectados por el número de UA del segundo y el tercer brotes (cuadro 3). La relación entre la longitud del tronco y su diámetro basal fue significativamente menor para las plantas de la procedencia Ñorquinco que para las plantas de las procedencias Pilolil y Quila-Quina al considerar el número de UA del segundo brote. La relación entre el diámetro basal de la planta y el número de nudos del tronco fue mayor para la procedencia Quillén 7 que para las procedencias Pilolil y Quila-Quina (cuadro 3).

Cuadro 3. Comparaciones de longitud, diámetro basal, número total de nudos del tronco y de los cocientes entre estas variables para plantas de roble de tres años mediante ANDEVA (prueba $F$ de Fisher). A: efectos de procedencia y del número de UA del segundo brote anual (1 ó 2). B: efectos de procedencia y del número de UA del tercer brote anual (1, 2 ó 3 ). ***: $P<0,001, * *: P<0,01, *: P<0,05$, ns: $P>0,05$.

ANOVA comparisons (Fisher's $F$ test) of trunk length, basal diameter and number of nodes and the ratios between these variables for plants of oak after three years of growth. A: effects of provenance and number of UA of second annual shoot (1 or 2). B: effects of provenance and number of UA of third annual shoot (1, 2 or 3). ***: $P<0.001, * *: P<0.01, *: P<0.05$, ns: $P>0.05$.

Prueba de Fisher

\begin{tabular}{llll}
\hline Procedencias & & \multicolumn{2}{l}{ UA en segundo brote } \\
& & $F$ & $P$
\end{tabular}

A

\begin{tabular}{lccrc} 
Longitud (L) & 2,8 & $*$ & 18,6 & $* * *$ \\
Diámetro (D) & 0,8 & $\mathrm{~ns}$ & 19,2 & $* * *$ \\
Nudos (N) & 1,7 & $\mathrm{~ns}$ & 17,1 & $* * *$ \\
L/D & 4,7 & $* *$ & 0,1 & $\mathrm{~ns}$ \\
L/N & 3,4 & $*$ & 8,1 & $* *$ \\
D/N & 3,4 & $*$ & 4,3 & $*$ \\
\hline
\end{tabular}

\begin{tabular}{llccc}
\hline B & \multicolumn{2}{c}{ Procedencias } & \multicolumn{2}{c}{ UA en tercer brote } \\
\hline Longitud & 1,6 & $\mathrm{~ns}$ & 5,6 & $* *$ \\
Diámetro & 2,7 & $*$ & 7,6 & $* *$ \\
Nudos & 0,5 & $\mathrm{~ns}$ & 14,1 & $* * *$ \\
$\mathrm{~L} / \mathrm{D}$ & 2,2 & $\mathrm{~ns}$ & 0,2 & $\mathrm{~ns}$ \\
$\mathrm{~L} / \mathrm{N}$ & 2,3 & $\mathrm{~ns}$ & 0,3 & $\mathrm{~ns}$ \\
$\mathrm{D} / \mathrm{N}$ & 3,4 & $*$ & 0,2 & $\mathrm{~ns}$ \\
\hline
\end{tabular}

\section{DISCUSIÓN Y CONCLUSIONES}

Como en otras especies de Nothofagus, el desarrollo del roble en los primeros años desde la germinación involucra notables variaciones morfológicas y arquitecturales vinculadas con la transición entre la fase de establecimiento de la plántula y la fase de inicio de formación del tronco. La mayoría de tales variaciones parecen producirse independientemente del sitio de procedencia de las semillas del que derivaron las plantas. Por ejemplo, el tamaño de los brotes que constituyen el eje vertical de cada planta aumenta entre el primero y el tercer años de desarrollo. El tamaño de los primeros dos brotes anuales es notablemente mayor para las plantas medidas en el presente estudio que para plantas de edad equivalente observadas a campo, dentro del área de distribución natural de esta especie (Raffaele et al. 1998). Esto sugiere que el crecimiento del roble en la fase de establecimiento puede incrementarse notablemente en condiciones de vivero. Dado el período de sequía que suele presentarse en el norte de la Patagonia argentina durante el verano, es probable que el suministro de agua en vivero sea la causa principal de esta diferencia.

Por otro lado, el elevado porcentaje de brotes con ápice muerto luego de su alargamiento tanto para los ejemplares medidos en el presente estudio como para aquellos observados a campo (Raffaele et al. 1998), indica que la muerte del ápice en los primeros brotes no se vincula necesariamente con la disponibilidad de agua. El crecimiento vigoroso que presenta el roble en vivero no impide que se produzca la muerte de un número variable de nudos, entrenudos y yemas distales de los brotes hacia el final de su período de alargamiento. Estudios previos han demostrado que el período del año en el que se produce el alargamiento de los brotes del tronco en Nothofagus spp. puede abarcar desde la primavera temprana (octubre) hasta las primeras semanas del otoño (abril), dependiendo de las condiciones ambientales y de la fase de desarrollo del individuo (Puntieri et al. 1998, Gallo et al. 2000). El hecho de que las primeras UA de los brotes del segundo y tercer años de desarrollo de los robles del presente estudio presentaran muerte de ápice con mayor frecuencia que las segundas o terceras UA podría reflejar la sensibilidad de los brotes en desarrollo a las heladas de primavera y verano temprano que caracterizan a la región cercana al lago Nahuel Huapi (Conti 1998), las que corresponderían con el período en el que se alarga la primera UA de los brotes. Esta hipótesis encuentra apoyo en un estudio anterior en el cual se observó alta sensibilidad a heladas tempranas (de fines del verano) en brotes con período de alargamiento prolongado de ejemplares de Nothofagus híbridos entre raulí y roble (Gallo et al. 2000).

En el presente estudio, el desarrollo de segundas o terceras UA en un mismo brote se produjo después de la muerte, aparentemente traumática, del ápice de la prime- 
ra UA. Tanto la segunda UA como la tercera UA derivaron de yemas axilares próximas a la porción muerta de la UA previa. Estas UA suplementarias continúan la línea de desarrollo vertical de la primera UA. En ocasiones se forman varias UA codominantes, de desarrollo vertical, a partir de dos o tres yemas de la UA previa. Es destacable el hecho de que los brotes conformados por más de una UA fueron, en promedio, más largos y gruesos y desarrollaron más nudos que aquellos conformados por una sola UA. Es decir, que la muerte del ápice de un brote de roble en desarrollo representa un beneficio en términos de la altura que puede alcanzar el tronco y del diámetro (y, presumiblemente, la resistencia mecánica) del último brote del tronco. En individuos juveniles de especies de Fagus y Quercus (las hayas y robles del Hemisferio Norte, evolutivamente emparentados con Nothofagus) los brotes suelen también estar conformados por varias UA, pero generadas a partir del meristema apical, sin muerte de ápice entre dos UA sucesivas. Al igual que en el caso descrito aquí, el desarrollo total de un brote en estas especies de Fagus y Quercus se incrementa con el número de UA constituyentes (Heuret et al. 2000, 2003, Nicolini et al. 2000). En el presente estudio no se observó la formación de segundas UA a partir de la yema terminal de un brote en desarrollo (es decir, sin la muerte del ápice de ese brote), un evento que parece ocurrir sólo excepcionalmente en las especies sudamericanas de $\mathrm{No}$ thofagus. Observaciones realizadas sobre esta y otras especies de Nothofagus indican que el desarrollo del tronco mediante sucesivos relevos no provoca deformaciones notables en el largo plazo, en oposición a lo observado en árboles con baja frecuencia de muerte apical (Kozlowski 1971).

Diferencias entre procedencias. En el presente ensayo no se registraron diferencias notables entre procedencias en cuanto al tamaño de las plantas al cabo de tres años de desarrollo. Las diferencias entre procedencias en la longitud del brote del primer año no se mantuvieron en los dos años siguientes. Los análisis realizados indican que la existencia de segundas o terceras UA en un mismo brote incrementan el desarrollo de las plantas en altura, diámetro basal y número de nudos del tronco. En la medida en que la tendencia a formar brotes con varias UA varíe con la procedencia de las plantas, podrían encontrarse diferencias en el tamaño de las plantas entre procedencias. Tomando en cuenta el número de UA por brote en la comparación entre procedencias, fue posible detectar diferencias que de otra forma habrían pasado inadvertidas. Por ejemplo, a igual número de UA por brote, las plantas de Quillén 7 desarrollan un tercer brote de mayor diámetro basal que las plantas de Quila-Quina. Asimismo, las relaciones entre diferentes medidas del tamaño de las plantas, como altura y diámetro basal, se modifican de acuerdo al número de UA de los brotes que las constituyen.
Las plantas de las procedencias de Quillén se diferenciaron de aquellas de las restantes procedencias en las menores frecuencias de muerte de ápice y de desarrollo de UA adicionales en el segundo año. En el tercer año se registraron altas frecuencias de muertes apicales para todas las procedencias, a pesar de lo cual la proporción de plantas de Quillén con UA adicionales fue menor que en plantas de las restantes procedencias, lo cual sugiere que la relación entre muerte apical y desarrollo de UA adicionales es menos directa de lo que podría suponerse. Dadas las diferencias de altitud entre las zonas de las cuales se recolectaron semillas, podría suponerse que Quillén presenta un período de crecimiento más corto y con mayor frecuencia de heladas que en las otras zonas consideradas aquí. Presiones de selección en Quillén habrían favorecido una menor probabilidad de rebrote luego de la muerte del ápice y mayor resistencia del ápice a las heladas, lo cual reduciría los efectos negativos de las bajas temperaturas. Estudios posteriores deberían (a) verificar los resultados del presente estudio en los sitios de recolección de las semillas, (b) analizar si la tendencia a la reducción en el número de UA por brote con la edad del tronco, observada para otras especies de Nothofagus (12), se expresa más tempranamente en poblaciones expuestas a períodos de crecimiento más cortos y (c) si las posibles diferencias en la expresión de ese gradiente dependen de factores genéticos.

\section{AGRADECIMIENTOS}

Agradecemos a Lucas Oudkerk por su trabajo en el establecimiento y mantenimiento del experimento. Este estudio fue financiado por la Universidad Nacional del Comahue (Proyecto B-704 UNC) y el CONICET (beca postdoctoral de J. Grosfeld y beca de postgrado de M. Stecconi).

\section{REFERENCIAS}

Barthélémy D, C Edelin, C, F Hallé. 1989. Some architectural aspects of tree aging. Ann. For. Sci. 46:194-198.

Barthélémy D, JG Puntieri, C Brion, E Raffaele, J Marino, P Martinez. 1999. Morfología de las unidades estructurales y modo de desarrollo básico de especies patagónicas de Nothofagus (Fagaceae). Bol. Soc. Argent. Bot. 34:29-38.

Barthélémy D, Y Caraglio, E Costes. 1997. Architecture, gradients morphogénétiques et âge physiologique chez les végétaux. In Bouchon J, P de Reffye, D Barthélémy eds. Modélisation et simulation de l'architecture des plantes. Paris, Francia. INRA Editions, Science Update. p. $89-136$.

Conti HA. 1998. Características climáticas de la Patagonia. In Correa MN ed. Flora Patagónica VIII (I). Buenos Aires, Argentina. INTA. p. 31-47. 
Costes E, PE Lauri, JM Lespinasse. 1995. Modélisation de la croissance et de la ramification chez quelques cultivars de Pommier. In Bouchon $\mathrm{J}$ ed. Architecture des arbres fruitiers et forestiers. Paris, Francia. INRA Editions, Les Colloques. p. 27-39.

Donoso C. 1993. Bosques Templados de Chile y Argentina. Santiago, Chile. Editorial Universitaria. 484 p.

Donoso C, L Gallo, P Donoso, MM Azpilicueta. 2004. Variación en Nothofagus obliqua (Mirb.) Oerst. (Roble, Coyán, Hualle o Pellín). In Donoso C, A. Premoli, L. Gallo, R Ipinza eds. Variaciones intraespecíficas en las especies arbóreas de los bosques templados de Chile y Argentina. Santiago, Chile. Universitaria. p. 79-113.

Emhart V, B Gutiérrez, R Ipinza, A Bello, M Navarrete, G Clasing. 2000. Establecimiento de huertos semilleros de Nothofagus. In Ipinza R, B Gutiérrez, V Emhart eds. Domesticación y mejora genética de raulí y roble. Valdivia, Chile. Universidad Austral de Chile. p. 283-295.

Gallo L, P Marchelli, P Crego, L Oudkerk, F Izquierdo, A Breitembücher, M González Peñalba, L Chauchard, L Maresca, Cuerpo de Guardaparques de los Parques Nacionales Lanín y Nahuel Huapi, U Mele. 2000. Distribución y variación genética en características seminales y adaptativas de poblaciones y progenies de raulí en Argentina. In Ipinza R, B Gutiérrez, V Emhart eds. Domesticación y mejora genética de raulí y roble. Valdivia, Chile. Universidad Austral de Chile. p. 133-155.

Gallo L, P Marchelli, P Crego. 2000. Dinámica de hibridación interespecífica entre raulí y roble pellín. In Ipinza R, B Gutiérrez, V Emhart eds. Domesticación y mejora genética de raulí y roble. Valdivia, Chile. Universidad Austral de Chile. p. 181-196.

Guédon Y, P Heuret, E Costes. 2003. Comparison methods for branching and axillary flowering sequences. J. Theor. Biol. 225:301-315.

Hallé F, RAA Oldeman, P Tomlinson. 1978. Tropical trees and forests. An architectural analysis. Berlin, Alemania. Springer-Verlag. $441 \mathrm{p}$.

Heuret P, D Barthélémy, E Nicolini, C Atger. 2000. Analyse des composantes de la croissance en hauteur et de la formation du tronc chez le chêne sessile, Quercus petraea (Matt.) Liebl. (Fagaceae) en sylviculture dynamique. Can. J. Bot. 78:361-373.

Heuret P, Y Guédon, N Gérard, D Barthélémy. 2003. Analysing branching pattern in plantations of young red oak trees (Quercus rubra L., Fagaceae). Ann. Bot. 91:479-492.

Ipinza R. 2000. Modelo básico de mejora genética. In Ipinza R, B Gutiérrez, V Emhart eds. Domesticación y mejora genética de raulí y roble. Valdivia, Chile. Universidad Austral de Chile. p. 215-235.
Kozlowski TT. 1971. Growth and development of trees, seed germination, ontogeny, and shoot growth. New York, USA. Academic Press. 443 p.

Nicolini E, D Barthélémy, P Heuret. 2000. Influence de la densité du couvert forestier sur le développement architectural de jeunes chênes sessiles, Quercus petraea (Matt.) Liebl. (Fagaceae), en régénération forestière. Can. J. Bot. 78:1531-1544.

Passo A, JG Puntieri, D Barthélémy. 2002. Trunk and mainbranch development in Nothofagus pumilio (Nothofagaceae): a retrospective analysis of tree growth based on the size and structure of its annual shoots. Can. J. Bot. 80:763-772.

Puntieri JG, D Barthélémy, P Martinez, E Raffaele, C Brion. 1998. Annual-shoot growth and branching patterns in Nothofagus dombeyi (Fagaceae). Can. J. Bot. 76:673-685.

Puntieri JG, E Raffaele, P Martinez, D Barthélémy, C Brion. 1999. Morphological and architectural features of young Nothofagus pumilio (Poepp. et Endl.) Krasser (Fagaceae) plants. Bot. J. Linn. Soc. 130:395-410.

Puntieri JG, MS Souza, D Barthélémy, C Brion, M Núñez, C Mazzini. 2000. Preformation, neoformation and shoot structure in Nothofagus dombeyi (Nothofagaceae). Can. J. Bot. 78:1044-1054.

Puntieri JG, C Brion, D Barthélémy, MS Souza. 2001. Variaciones en el tamaño y el contenido de las yemas de Nothofagus pumilio y Nothofagus dombeyi (Fagaceae). Darwiniana 39:1-10.

Puntieri JG, M Damascos, MS Souza. 2001. Tendencias ontogenéticas en el tamaño y la forma de las hojas de Nothofagus pumilio (Poepp. et Endl.) Krasser (Fagaceae). Ecología Austral 11:105-114.

Puntieri JG, D Barthélémy, C Mazzini, C Brion. 2002a. Periods of organogenesis in shoots of Nothofagus dombeyi (Mirb.) Oersted (Nothofagaceae). Ann. Bot. 89:115-124.

Puntieri JG, M Stecconi, D Barthélémy. 2002b. Preformation and neoformation in shoots of Nothofagus antarctica (G. Forster) Oerst. (Nothofagaceae) shrubs from northern Patagonia. Ann. Bot. 89:665-673.

Raffaele E, JG Puntieri, P Martinez, J Marino, C Brion, D Barthélémy. 1998. Comparative morphology of annual shoots in seedlings of five Nothofagus species from Argentinean Patagonia. C. R. Acad. Sci. Paris 321:305-311.

Sokal RR, FJ Rohlf. 1981. Biometry. Second edition. New York, USA. Freeman. 859 p.

Souza MS, JG Puntieri, D Barthélémy, C Brion. 2000. Bud content and its relation to shoot size and structure in Nothofagus pumilio (Poepp. et Endl.) Krasser (Nothofagaceae). Ann. Bot. 85:547-555.

Stecconi M, JG Puntieri, D Barthélémy. 2000. Annual shoot growth in Nothofagus antarctica (G. Forster) Oersted (Nothofagaceae) from northern Patagonia. Trees 14:289-296. 